\title{
Tricolor Light-Emitting Diode Dot Matrix Display System with Audio Output
}

\author{
Grantham K. H. Pang, Member, IEEE, Chi-Ho Chan, and Thomas T. O. Kwan
}

\begin{abstract}
In this paper, a novel tricolor dot matrix display system in which all the light-emitting diodes (LEDs) are modulated and encoded with audio information is presented. The novelty is on the concurrent use of the tricolor LED display for simultaneous visual message display and audio signal transmission in a free-space optical link. This is a new generation of tricolor dot matrix displays where, in addition to the usual function of LED display, they concurrently provide audio broadcasting through the visible light rays transmitted by the display panel or assembly.The system is comprised of a tricolor dot matrix display panel with an interface circuit to a computer, an audio signal transmitter, and a receiver. It also comprises an executive program that runs on the computer for the display control of characters, decorative pattern or messages on the display panel. The receiver is designed to demodulate the optically transmitted audio information, and reproduce the messages with the speaker. For modulating emission of the LEDs, an oscillator is used to vary the frequency of on/off periods of the LEDs. The frequency of flicker is high enough to be indistinguishable by human eye and, hence, display appears to be constantly illuminated.
\end{abstract}

Index Terms-Audio communication, light-emitting diodes, tricolor display.

\section{INTRODUCTION}

$\mathbf{T}$ HIS PAPER relates to a tricolor dot matrix display that provides visual signals for transmitting audio information to a receiver located some distance away from the display system. The basic idea is based on the fact that light-emitting diodes (LEDs) are semiconductor devices and are capable of fast switching with the addition of appropriate electronics. That is, the visible light emitted by the LEDs can be modulated and encoded with audio information. Therefore, in addition to their normal function of indication and illumination, LEDs can be used as a communication device for the transmission of audio information. Optical signals have been used for communication and information processing in the field of fiber optics for many years. In this paper, a different use of optical signals for communication is described. As for dot matrix display, the basic working principle is that information is encoded in each row. Each glowing row will transmit a portion of the

Paper MSDAD-S 00-48, presented at the 2000 Industry Applications Society Annual Meeting, Rome, Italy, October 8-12, and approved for publication in the IEEE TRANSACTIONS ON INDUSTRY APPLICATIONS by the Industrial Automation and Control Committee of the IEEE Industry Applications Society. Manuscript submitted for review October 15, 2000 and released for publication January 8, 2001.

The authors are with the Department of Electrical and Electronic Engineering, The University of Hong Kong, Hong Kong, (e-mail: gpang@hkueee.hku.hk; chichan@eee.hku.hk)

Publisher Item Identifier S 0093-9994(01)02498-7. information. As long as the receiver can cover all rows, the complete information is received as if it is transmitted from a single source.

There has been rapid advance in the LED technology in recent years. Today, visible light LEDs can reach a luminous efficacy beyond $15 \mathrm{~lm} / \mathrm{W}$, which is typical for incandescent lamps [1]. Even higher performance can be achieved in the yellow-orange spectral range. The AlInGaP technology has produced high brightness LEDs from red-orange to green [2], [3]. This advancement has led to the production of large-area full-color LED displays for outdoors, as well as many new applications in the industry. Current studies [4] have shown that using LED instead of incandescent light in traffic light has many advantages, including money and energy saving, and with much easier maintenance. Recent research has shown a new idea on the dual use of the LED-based signals [5]-[8]. Due to the fast switching capability of LEDs, information can be transmitted optically to a photodiode-based receiver in a wireless way.

Pang et al. [6] describe the idea, design, evaluation, and implementation of LED wireless communication systems. Both analog audio broadcast and digital data communication systems are covered. An intelligent traffic light was fabricated, and evaluation experiments were conducted. The viewing angle (half power) is found to be $30^{\circ}$. For digital signal, the transmission rate can reach $500 \mathrm{~kb} / \mathrm{s}$, and the throughput is around 2000 byte/s. For an optical power at the receiver of $0.8 \mu \mathrm{W}$, the bit error rate $(\mathrm{BER})$ is around $9 \times 10^{-8}$. This paper focuses on its use for audio transmission through a dot matrix display.

LEDs have been used as basic components in dot matrix display for years. Some researchers have worked on improved LED display that includes a printed circuit board, a reflector, and one or more LED chips [9]. The parabolic reflector surrounding the surface is used to collimate light waves emitted by LEDs along the axis of parabola. However, no work has been reported regarding its use for simultaneous audio transmission. The work reported in this paper differs from the use of infrared (IR) radiation as a medium for short-range wireless communications [10], [11]. Currently, IR links and local area networks (LANs) are available. IR transceivers for use as IR data links are commercially available at economic prices. Comparison between the IR and other media such as radio and microwave is given in [12]. Somehow, there has been very little work on the use of visible light as a communication medium. Recent availability of high brightness LEDs makes the visible light medium even more feasible now. Essentially, all products with visible LED components (for example, a LED traffic signal head) can be turned into an information beacon. 


\section{SYSTEM DESCRIPTION}

This paper describes the concurrent use of tricolor LED display for simultaneous signaling and broadcasting of audio information. It relates to a dot matrix tricolor display panel, or any multicolor LED display assembly for providing a visual signal in the form of light rays, and for using the light rays to transmit information. It is a very versatile dot matrix display of characters (Chinese or English), picture, graphics, or any decorative pattern. The LED display is controlled by a computer through a hardware interface. An executive program is used for the visual display and control of characters, decorative patterns, or messages on the display panel. The system is comprised of a transmitter and a receiver. The transmitter consists of an audio signal source that provides the audio information to be transmitted by the display assembly. The receiver consists of a lens system for focusing the light waves from the LEDs to the photo detector. The optical link employs intensity modulation of LEDs with direct detection. With appropriate electronics, the information in the light ray is demodulated to detect the audio signal, which is reproduced with a speaker for the broadcasting and announcement of the audio messages. The advantages of this circuit over single-color LED displays are as follows:

1) Tricolor Display-As illustrated in Fig. 5, the LED would show one color when only the column signal one is pulled high, and show a second color when the column signal two is high. If both column signals one and two are high, it will show a third color. As tricolor displays are used, the background of the display can also be lighted up using a color which is different from the main character to be displayed. Yet, the LEDs from the same row will be transmitting the same information. So for the receiver, there will always be LED lit up in each row, and so it will receive information from each row at any time, and no information would be lost. Also, the pattern or graphics displayed will be more attractive and flexible as more colors can be added for the display in each panel.

2) Brightness - With all LEDs glowing, the brightness of the transmitter is dramatically enhanced. This makes the audio transmission more effective and immune to noise as the attenuation in the medium becomes negligibly small for line-of-sight (LOS) reception. In addition, the receiver's distance from the transmitter can be increased.

3) Lowercase Characters-As the background is lighted up, the characters displayed will not be confined to the set that does not have an inactive row. In particular, lowercase characters (e.g., "i") can be displayed without loss of any information during transmission.

4) One-Column Coverage is Sufficient-As virtually all the LEDs are lighted up in the course of pattern display, and each LED in a single row is transmitting exactly the same information, the receiver in principle needs to locate one column to receive all information. This will relax the previous restriction that the lens used in the receiver should cover all columns.

A block diagram of the tricolor light emitting diode dot matrix display system is shown in Fig. 1. The visible light from

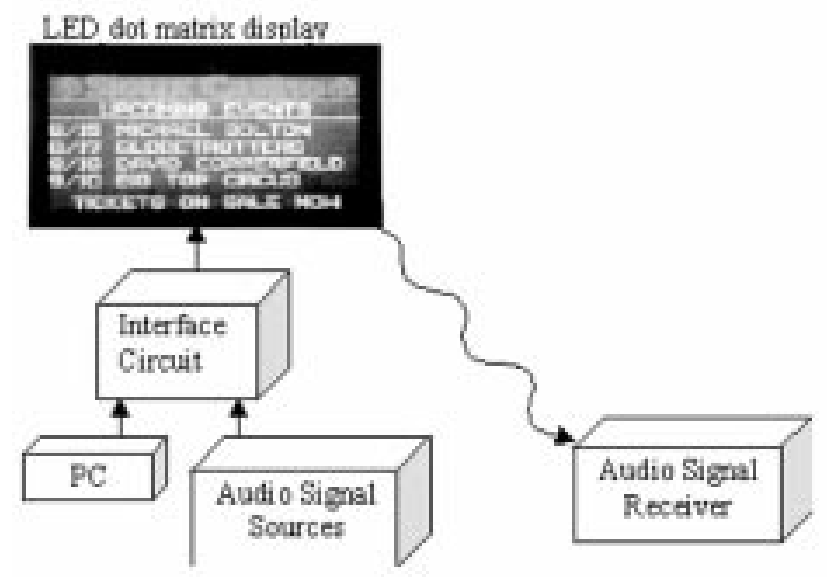

Fig. 1. Block diagram of the tricolor LED dot matrix display system, an interface circuit which links the display to a computer, an audio signal transmitter, and the companion receiver.

the LED display panel is used for transmitting information, in addition to providing a visual signal. The display is linked to a computer through an interface circuit, which provides the control of characters to display and the addition of audio signals.

The LED display has a plurality of LEDs for transmitting an optical signal. The display, which acts as a transmitter, includes a voltage-controlled oscillator (VCO) for varying the switching frequency of light emitting diodes on the dot matrix display. The on/off frequency is high enough such that the perceivable light appears to be constantly illuminated to the human eye. The present system has achieved to modulate the visual light rays emitted by the LEDs for the transmission of audio information. As shown in Fig. 2, the transmitter provides a modulated audio signal from a cassette tape player or CD player by frequency modulation (FM). The receiver makes use of a photo-detector to detect high frequency variations in the light flux. This detected signal is passed to appropriate amplifying and detecting stages to reproduce audio signal transmitted. Both the transmitter and the receiver require low power supply, which increases transportability of the system. As the majority of the circuitry is TTL based, power supply requirements are in the vicinity of five volts.

Referring to Fig. 2, the audio signal from the cassette tape or $\mathrm{CD}$ player has small amplitude. The audio amplifier is used to amplify the weak audio signal, and to shift the average voltage level of the audio signal to an appropriate level so that the signal is suitable for input to the voltage-controlled oscillator (VCO). An incoming audio signal modulates the VCO and generates FM signal at the output, according to the change in voltage level of the signal. A square-wave VCO is used instead of sine wave because there are only two states (ON and OFF) for the LEDs. The carrier frequency is set at $100 \mathrm{KHz}$ with a maximum frequency deviation of $\pm 50 \mathrm{KHz}$. The modulated signal is transmitted by the switching of the LEDs, through a driving circuit.

A block diagram representation of the receiver design is shown in Fig. 3. The circuit of the receiver is divided into eight portions. The photo-detector is used to detect a modulated light signal from the transmitter and convert the signal into an electrical signal. The limiting pre-amplifier is used to amplify 


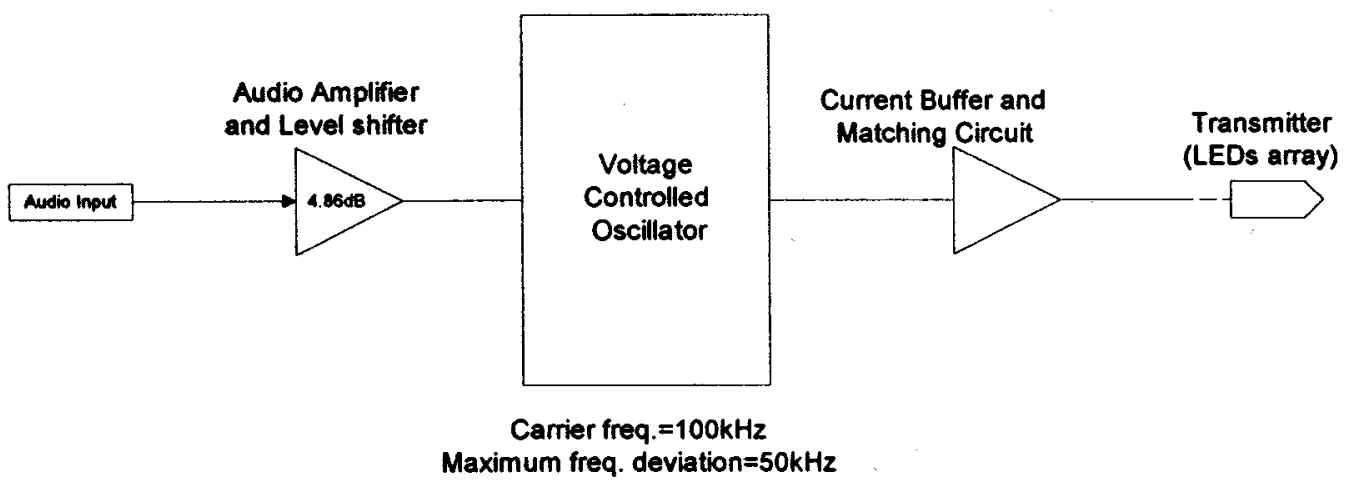

Fig. 2. Block diagram representation of the schematic diagram of the audio signal transmitter.

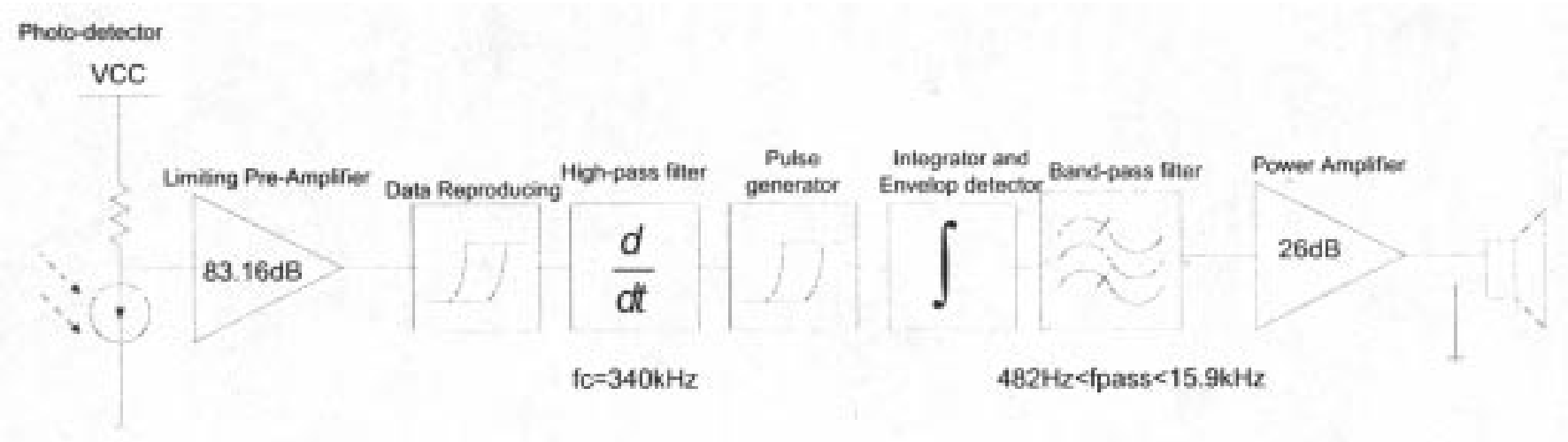

Fig. 3. Block diagram representation of the receiver.

the electrical signal from the photo-detector for the next stage. Pulse shaping and detecting circuits are used to reconstruct the square wave. Square wave pulses from previous output stages are differentiated, and its negative-trailing edges are clipped when transmitted through a diode. Next, a pulse generator is used to convert the pulses from the differentiator into sharp pulses, to be used by the integrator and envelope detector in the next stage for the demodulation of the signal. The band-pass filter is then used to smooth out the distortions from the integrator and envelope detector to produce an appropriate waveform. Finally, an audio power amplifier is used to amplify the weak signal from the band-pass filter, so that the audio signal would be comfortable for hearing. A speaker, headphone or ear jack can be used for the delivery of the audio messages or music sent from the transmitter. The receiver can be battery powered, or powered by any electrical system.

The interface circuit, which is connected to the parallel port of the computer, is shown in Fig. 4. There are seven parts for this circuit. Below is a description of the major components:

2) Row Counter Decode System-The counter is driven by the information signal, and a decoder would select the row for display.

3) Column Counter Decode System-This contains a counter, driven by a fast oscillator, and a decoder for color/column selection.

4) Pattern Storage System-Static random access memory (SRAM) is used to store the desired pattern for each row and column color of the displayed characters.
5) Address Comparison Unit-It compares the system-generated address against the address sent out by the computer, and contains a bit comparator and a register. This register holds the data until the system is ready to write the data to the SRAM.

6) Status Indicator-It contains a D-type flip-flop with asynchronous inputs of Preset and Clear. The noninverted flip-flop output pin is used for indication of a successful write. On such occasion, this pin will be set high, and will enable the computer to send the next data.

7) LED Display Unit-It temporarily stores the LED information and holds the LEDs in place. This unit contains registers to isolate the LEDs with the circuit. Fig. 5 gives a schematic of a tricolor LED dot matrix array. In order for a LED to be on, the corresponding row signal should be low, and the associated column signal should be high.

8) Parallel Port-This is the interface to the hardware electronics. Data and control signals are sent out from this port, and status information is delivered to the computer via this port.

\section{SPecial Features of the Tricolor Display}

\section{A. Timing Signal for Information Update}

There are two main techniques in this design of the tricolor display.

1) Multiplexing of row and column timing signals for modulation: There are two timing signals in the system. The first one is the information signal that contains the modulated carrier 


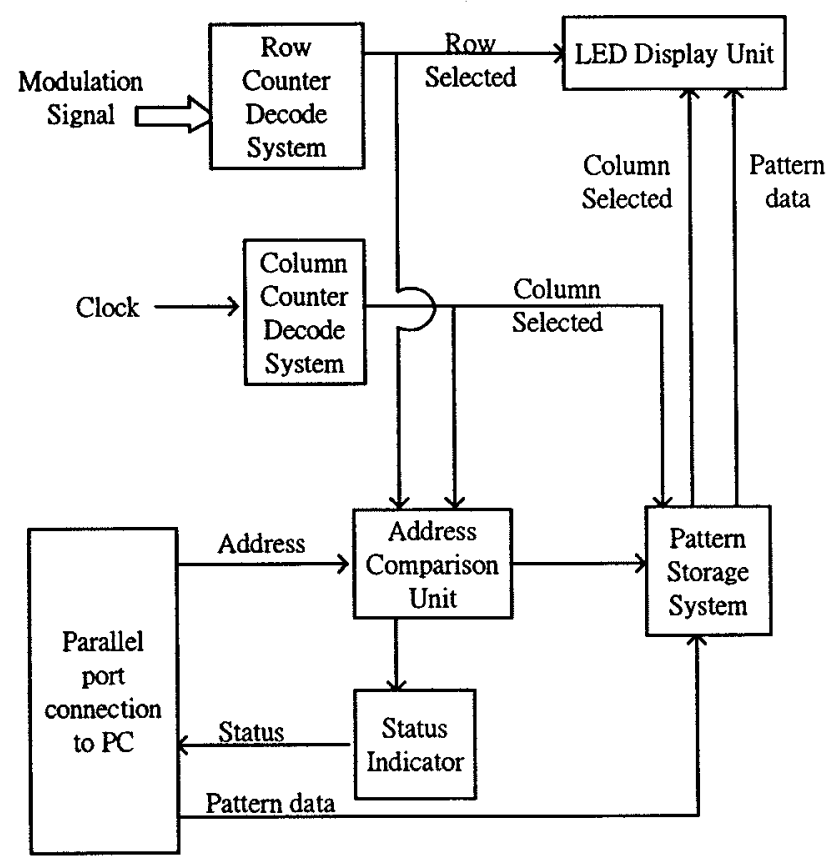

Fig. 4. Circuit diagram of the interface design.

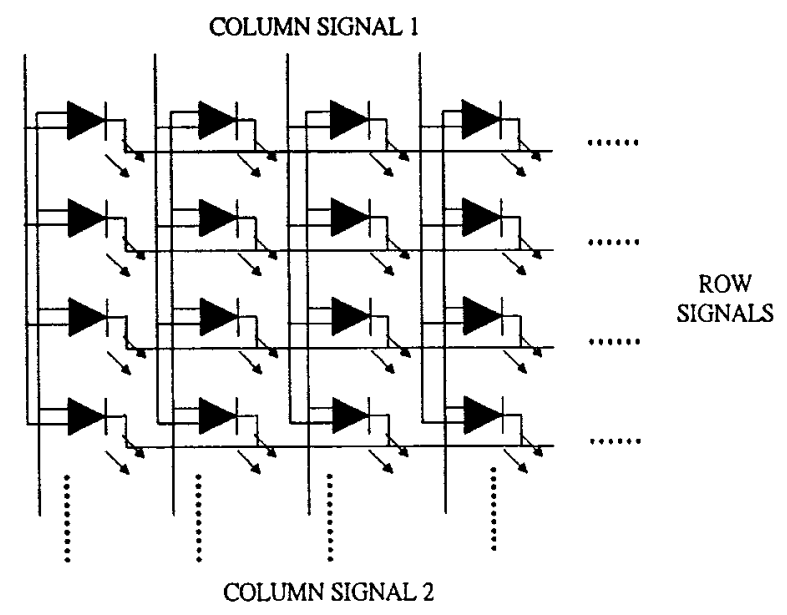

Fig. 5. Schematic of a tricolor dot matrix display using light emitting diodes.

frequency. Whenever the information signal changes, the active row will also be changed. In short, the row represents a bit of information. A $100-\mathrm{KHz}$ information signal is used in the current implementation. In addition, an audio signal is modulated into this signal that occupies a frequency range of $(100 \pm 44.1)$ $\mathrm{kHz}$.

The second timing signal for the column is derived from an oscillator. In this implementation, a 12-MHz oscillator frequency is used. This signal is used to switch between different columns (i.e., different colors in different characters). It must switch at a much higher rate than the information signal, so that before the information signal is changed (i.e., before the active row is switched), all the columns should have been scanned at least once and updated. Otherwise, some columns will not be lit as expected.
While the row signal is changed at a frequency of the information signal (e.g., $100 \mathrm{kHz}$ ), the column signal is changed at a higher frequency of about $12 \mathrm{MHz}$. However, this $12 \mathrm{MHz}$ will be divided by the counter (since it acts as a frequency divider also) and the counter output is, in fact, running at $6 \mathrm{MHz}$ only. In addition, only half of the $100-\mathrm{kHz}$ period is used for column selection. Therefore, during the period that a row is active, the number of times the column address is changed is

$$
\frac{6 \mathrm{MHz}}{100 \mathrm{kHz}} * \frac{1}{2}=30 \text {. }
$$

Each column has about three times to be updated. Actual number of times depends on the information signal that can be changed from time carryout time.

2) Updating is carried out during the period when the LEDs are off: This circuit is synchronized with the information signal at any time, including the time when the patterns are updated. The technique is to carry out the updating when the row is not active. Then no noise will be heard because no data will be transmitted during the pattern update process.

\section{B. Pattern Update}

The first part of the address for the data is from the information signal that specifies the row at which the LEDs are active. The other part is from the $12-\mathrm{MHz}$ oscillator that specifies the column for which data is to be latched to the display register. The data address is then loaded in the SRAM for data request. After the data is retrieved, it is loaded in the appropriate display register, and is displayed.

\section{Software for Display and Pattern Update}

A software program is used for the display control and pattern update. For the write cycle, data is first put onto the bus and latched. The corresponding address is than loaded in the same bus and compared with the system-generated address. If they are matched, data is written in the SRAM at the corresponding address. A signal is sent back to the computer, indicating a successful write.

Display effects such as scrolling and running can be carried out as follows. Suppose there is a five-character buffer, with the first four in the active display window (i.e., data contained will be transmitted to the dot matrix display). The fifth character buffer is introduced to accommodate the next character to be displayed, because as the text is scrolled, the data from the fifth character is required. Source codes have been written to shift the data logically to the left to generate the scrolling effect.

A special character data set used for the tricolor display has been used. The $6 \times 6$ characters are used on an $8 \times 8$ display so that the characters can be clearly distinguished when they are put side-by-side. The residue LEDs form a border around the character, which are displayed as background color.

\section{ApPlications of the LED Dot Matrix Display}

The tricolor display with audio output can be applied to a large variety of applications. As shown in Fig. 6, the LED display system can include a table, a target (such as a person standing in a room in front of the table), an LED display, and 


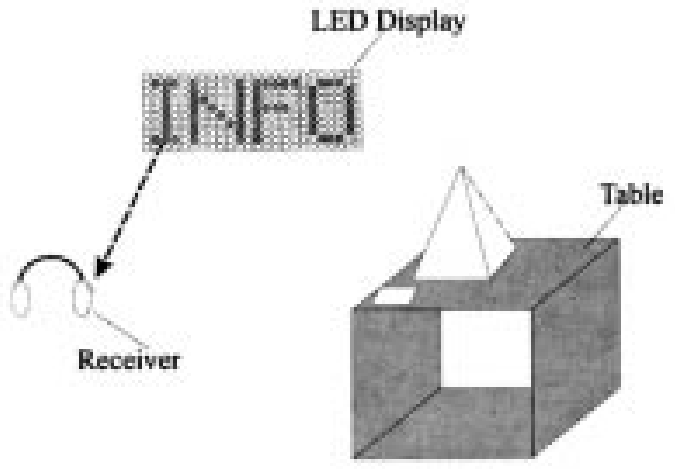

Fig. 6. A perspective view of the present system in a museum environment.

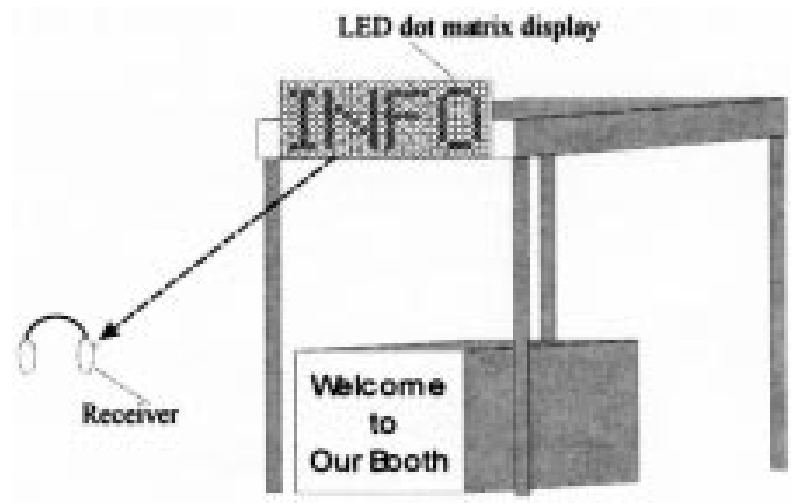

Fig. 7. A perspective view of the present system in an exhibition hall environment.

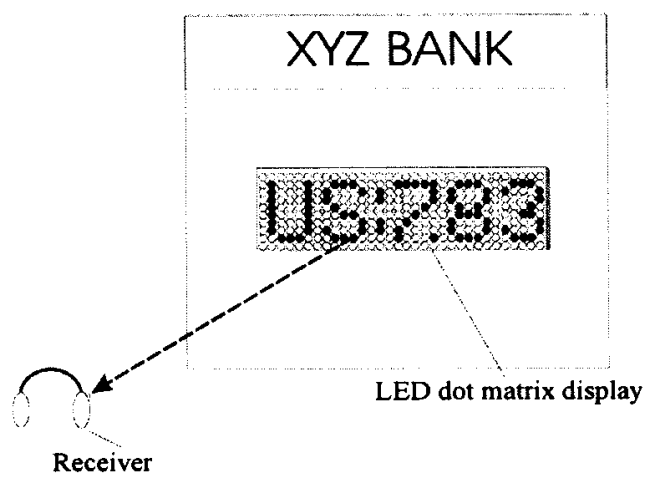

Fig. 8. A perspective view of the present system used outside a business or financial institution.

a receiver. Typically, an exhibit, or any physical object, is placed on the table. The indoor environment can be a room in a museum. The guest holding the portable receiver with an ear jack can listen to the audio messages transmitted through the LEDs of the dot matrix display. A quiet atmosphere can be maintained while the guests stroll around in the room. A major advantage over a conventional broadcasting system is that an individual with a receiver has the freedom of choice in receiving specific messages without hearing any unwanted announcement, music, or commercials.

In another application in an exhibition hall, as shown in Fig. 7, the information on an individual product or specific announcement of the company can be broadcast via the LED dot matrix display. When the visitor is looking at display panel, he or she is

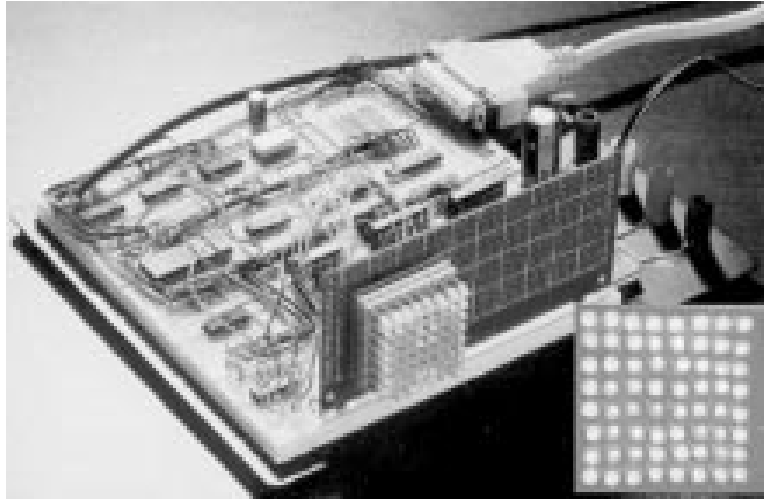

Fig. 9. Photograph of the tricolor LED dot matrix display.

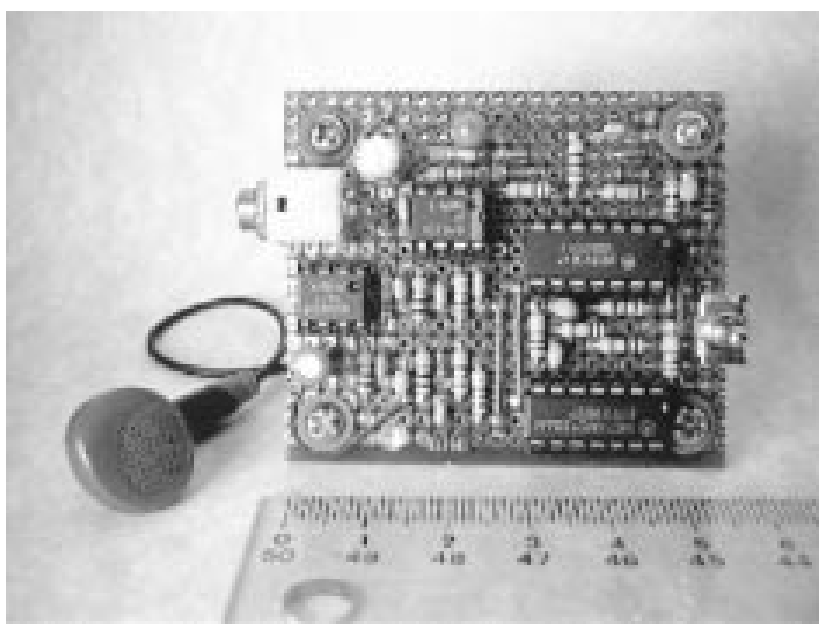

Fig. 10. Photograph of the receiver.

automatically in the line of sight and, thus, receives audio messages about the specific announcement. Thus, the sound level of the exhibition hall can be minimized.

In front of any business or commercial installations, it is common to find LED dot matrix displays which visually show some messages or announcements (Fig. 8). The light emitted from the LEDs in the display can be used to optically transmit audio information to the user with a receiver located some distance away from the display system. Thus, the visual display can also transmit audio information simultaneously. Hence, all commercial billboards can be used to transmit additional information, such as daily specials, discount, latest attractions or any merchandise, reservation telephone number, etc., for advertisement purposes.

\section{IMPLEMENTATION AND RESULTS}

The tricolor display system described in this paper has been implemented in the Industrial Automation Laboratory, The University of Hong Kong. Figs. 9 and 10 are photographs of the tricolor dot matrix display and receiver implemented, respectively. The display consists of an $8 \times 8$ LED dot matrix. The receiver was placed at two meters away from the display, and satisfactory audio output was obtained. Without a $50 \mathrm{~mm}$ focusing lens in front of the photodiode, the distance has reduced to $40 \mathrm{~cm}$. It should be noted that the type of LED used for the construction 
TABLE I

SPECIFICATIONS OF THE TRICOLOR DOT MATRIX DISPLAY

\begin{tabular}{l|l}
\hline Color & red, green, orange \\
\hline Construction & 64 tricolor LEDs \\
\hline Size & $4 \mathrm{~cm}$ square \\
\hline Nominal usage & $5 \mathrm{~V} ; 0.24 \mathrm{~A}$ \\
\hline $\begin{array}{l}\text { Nominal power } \\
\text { consumption }\end{array}$ & $1.2 \mathrm{~W}$ \\
\hline Luminous intensity & $180 \mathrm{~cd}$ \\
\hline $\begin{array}{l}\text { Viewing angle } 2 \theta_{1 / 2} \\
\text { (half power) }\end{array}$ & $80 \mathrm{degrees}$ \\
\hline $\begin{array}{l}\text { Distance for audio } \\
\text { transmission } \\
\text { (indoors) }\end{array}$ & $40 \mathrm{~cm}$ (no lens); \\
\hline
\end{tabular}

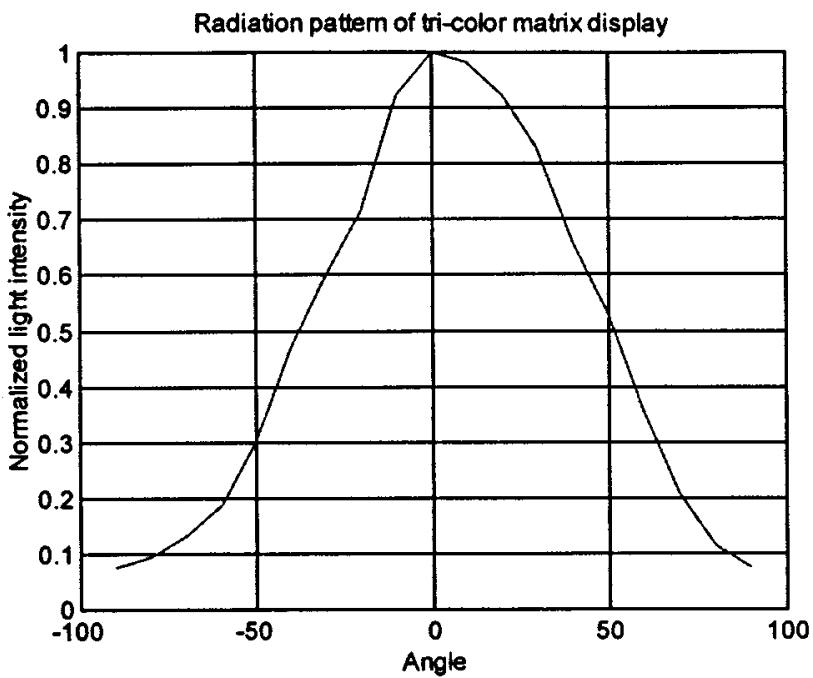

Fig. 11. Radiation pattern of the transmitter.

of the implemented dot matrix display is commonly available. Large audio transmission range can be achieved by employing high brightness LEDs [1], [3]. It is found that audio signal transmission can also be achieved outdoors.

The specifications of the tricolor dot matrix display is given in Table I.

The viewing angle of the display is around $80^{\circ}$. Fig. 11 shows the radiation pattern of the panel. An HP Audio Analyzer has been used to provide audio measurement for the visible light LED audio broadcasting panel. It has a low distortion signal source with a signal analyzer. In a measurement on the signal-tonoise ratio, the result is shown in Fig. 12. The distortion measurement on signals from 237 to $10 \mathrm{kHz}$ is shown in Fig. 13. The frequency response characteristic of the system is shown in Fig. 14. The system has been implemented using very low-cost electronic components. The relatively poor frequency response and distortion is only a limitation of the circuit hardware.

As the principle of audio transmission in our system is based on intensity modulation, the ambient lightings and light scattering will not cause deterioration in the performance of our system. The above has been verified in experiments. The system

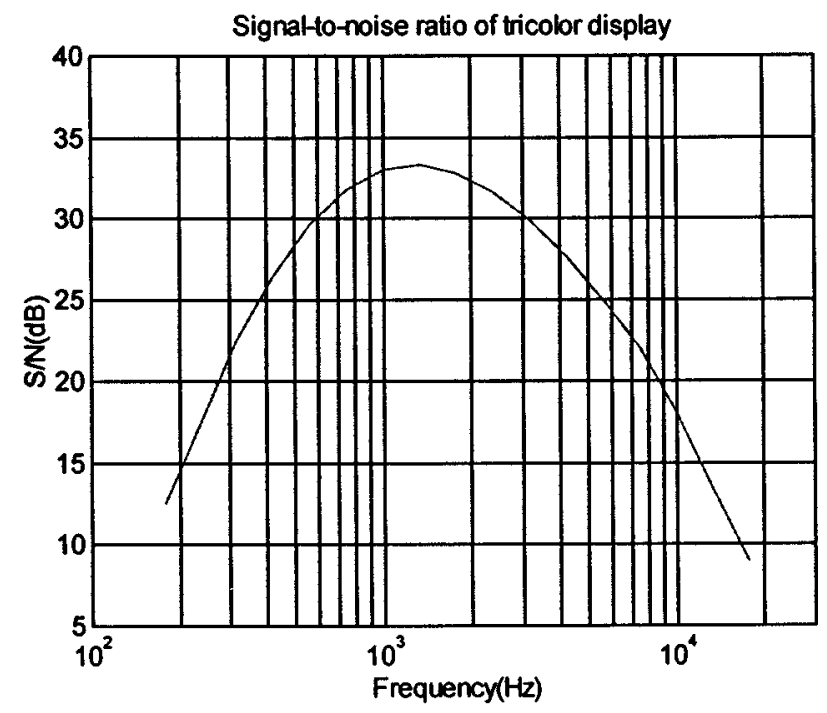

Fig. 12. Measurement on the signal-to-noise ratio.

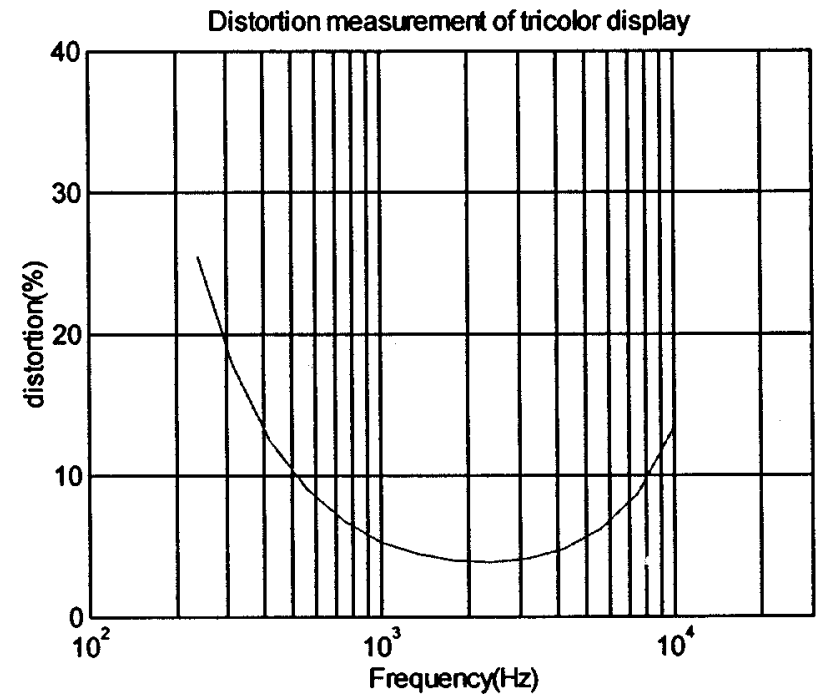

Fig. 13. Distortion measurement.
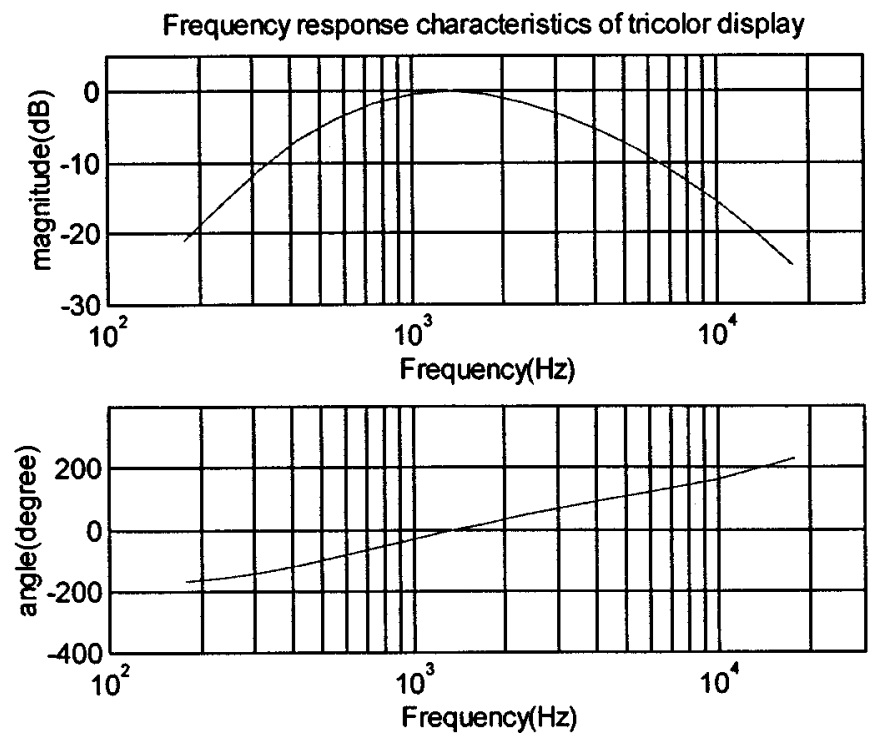

Fig. 14. Frequency response characteristic of the system. 
relies on direct line of sight between the transmitter and the receiver. Hence, crosstalk would affect the audio quality on reception.

In a typical application, the relative positions of the audio signal broadcasting system and the receiver would be stationary. Therefore, there should be no apparent increase or decrease in the frequency of light. The performance of the system is not affected by the Doppler effect.

\section{CONCLUSIONS}

A tricolor LED dot matrix display system, which provides a visual signal and uses the visual light rays for transmitting audio messages to a remotely located target, is described. The display characters (Chinese or English, uppercase or lowercase), picture, graphics, or any decorative pattern can be conveniently implemented. Scrolling of the pattern on the LED display panel is allowed. During pattern updates, no noise is received at the receiver. The receiver needs to cover only one column in order to recover all information transmitted from the dot matrix display. A software program is used to control the LED display in each row and column of the display panel to display the desired pattern.

The LED dot matrix display described in this paper belongs to a new generation of dot matrix displays, as the visual light emitted from the display contains audio messages. It has long been realized that visible light has the potential to be modulated and used as a communication channel with high entropy. This application makes use of free space as a communication medium, and the receiver is required to be in LOS with the transmitter. It should be noted that the transmitter provides easy target for the line-of-sight reception by the receiver. This is because the LEDs, being on all times, are also indicators of the locations of the transmitter. This development can lead to many new applications in the industry. With diverse commercial and industrial applications, the experimental results in this paper have demonstrated that the proposed idea is totally feasible.

\section{REFERENCES}

[1] G. B. Stringfellow and M. G. Craford, "High brightness light emitting diodes," in Semiconductors and Semimetals. New York: Academic, 1997, vol. 48

[2] M. G. Craford, "LEDs challenge the incandescents," IEEE Circuits Devices Mag., vol. 8, pp. 24-29, Sept. 1992.

[3] K. Werner, "Higher visibility for LEDs," IEEE Spectr., vol. vol. 31, pp. 30-39, July 1994

[4] J. O'Connell, "The Philadelphia story," Traffic Technol. Int., pp. 106-110, Aug./Sept. 1997.

[5] P. P. Smyth et al., "Optical wireless-A prognosis," Proc. Wireless Data Transmission, vol. 2601, pp. 212-225, Oct. 23-25, 1995.

[6] G. Pang et al., "LED traffic lights as a communication device," in Proc. Int. Conf. Intelligent Transportation Systems, Tokyo, Japan, Oct. 5-8, 1999, pp. 788-793.

[7] G. Pang et al., "Dual use of LEDs: Signaling and communications in ITS," presented at the 5th World Congress on Intelligent Transport Systems, Seoul, Korea, Oct. 12-16, 1998,

[8] G. Pang et al., "Optical wireless based on high brightness visible LEDs," in Conf. Rec. IEEE-IAS Annu. Meeting, Phoenix, AZ, Oct. 3-7, 1999, pp. 1693-1699.
[9] LED Products Data Book, Dialight, Manasquan, NJ.

[10] T. S. Chu and M. J. Gans, "High speed infrared local wirelesss communication," IEEE Comm. Mag., vol. 35, pp. 4-10, Aug. 1997.

[11] M. Meyer, "Infrared LEDs," Compound Semicond., pp. 39-40, May/June 1996.

[12] J. M. Kahn and J. R. Barry, "Wireless infrared communications," Proc. IEEE, vol. 85, pp. 265-298, Feb. 1997.

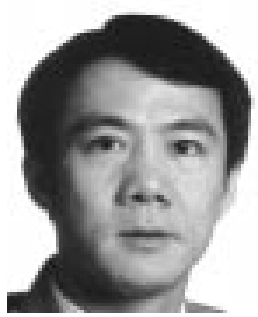

Grantham K. H. Pang (S'84-M'86) received the $\mathrm{Ph} . \mathrm{D}$. degree from the University of Cambridge, Cambridge, U.K., in 1986.

$\mathrm{He}$ was with the Department of Electrical and Computer Engineering, University of Waterloo, Waterloo, ON, Canada, from 1986 to 1996. In 1996, he joined the Department of Electrical and Electronic Engineering, The University of Hong Kong, Hong Kong. In 1994, he was a Senior Visiting Researcher at Hitachi Research Laboratory, Japan. He has acted as a Consultant to many companies, including Mitsubishi Electric Corporation, Japan, and Northern Telecom and Imperial Oil Ltd. in Canada. He is an Editor of the International Journal of Intelligent Control and Systems. He is also an Editor of Control and Computers, published by the International Association of Science and Technology for Development (IASTED). Since 1988, he has authored more than 80 published technical papers, and has authored or coauthored three books. His research interests include expert systems for control system design, intelligent control, intelligent transportation system, neural networks, control theory, and computer-aided design.

Dr. Pang was the Organizing Chair of the 1996 IEEE Symposium on Computer-Aided Control System Design. He was appointed by the President of the IEEE Control Systems Society as the Chair of the Technical Committee on Computer-Aided Control System Design (1993-1995). In 1989, he was awarded the ICI Prize for authorship of the best paper on the application of the theory of control published in the Transactions of Institute of Measurement and Control. $\mathrm{He}$ is a Chartered Electrical Engineer in Hong Kong and a member of the Instituion of Electrical Engineers, U.K., and Hong Kong Institution of Engineers.

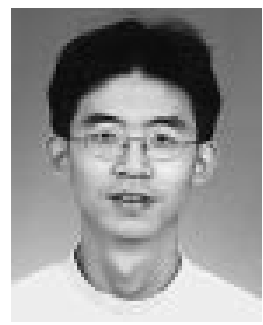

Chi-Ho Chan was born in Hong Kong. He received the B.Eng. degree from the University of Bradford, Bradford, U.K., and the M.Phil degree from The University of Hong Kong, Hong Kong, in 1997 and 2000, respectively, both in electrical and electronic engineering.

$\mathrm{He}$ is an Engineer on the Fabric Inspection Project at The University of Hong Kong, which is funded by the government and industrial support funds. His major research interests include image processing and analysis.

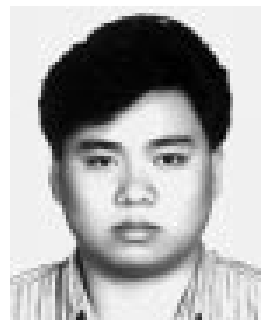

Thomas T. O. Kwan was born in Hong Kong. He received the H.C. degree in electronic engineering from The Hong Kong Polytechnic University, Hong Kong.

$\mathrm{He}$ was an Application Engineer with Hualon Microelectronics Corporation, working in ASIC development. In 1997, he joined The University of Hong Kong, Hong Kong. He is currently working as a Technician in the Industrial Automation Research Laboratory, Department of Electrical and Electronic Engineering. Prior to this, he provided services to consumer electronics companies for designing microcontroller units. His research interests include visible light communication, microcontroller unit development environment (3rd-Force), and intelligent transport systems. 